\title{
Spin transfer in diffusive ferromagnet-normal metal systems with spin-flip scattering
}

\author{
Alexey A. Kovalev, ${ }^{1}$ Arne Brataas, ${ }^{2, *}$ and Gerrit E. W. Bauer ${ }^{1}$ \\ ${ }^{1}$ Department of NanoScience, Delft University of Technology, 2628 CJ Delft, The Netherlands \\ ${ }^{2}$ Harvard University, Lyman Laboratory of Physics, Cambridge, Massachusetts 02138
}

(Received 18 July 2002; revised manuscript received 30 September 2002; published 31 December 2002)

\begin{abstract}
The spin transfer in biased disordered ferromagnet $(\mathrm{F})$-normal metal $(\mathrm{N})$ systems is calculated by the diffusion equation. For F1-N2-F2 and N1-F1-N2-F2-N3 spin valves, the effect of spin-flip processes in the normal metal and ferromagnet parts are obtained analytically. Spin-flip in the center metal N2 reduces the spin-transfer, whereas spin-flip in the outer normal metals N1 and N3 can increase it by effectively enhancing the spin polarization of the device.
\end{abstract}

DOI: 10.1103/PhysRevB.66.224424

PACS number(s): 72.25.Ba, 75.70.Pa, 75.60.Jk, 72.25.Rb

\section{INTRODUCTION}

A spin-polarized electric current flowing through magnetic multilayers with canted magnetizations produces torques on the magnetic moments of the ferromagnets. ${ }^{1,2}$ The effect is inverse to the giant magnetoresistance, in which a current is affected by the relative orientation of the magnetization directions. The spin-current-induced magnetization torque arises from an interaction between conduction electron spins and the magnetic order parameter, transferring angular momentum between ferromagnetic layers, hence the name "spin transfer." The observed asymmetry of the switching with respect to the direction of current flow in the magnetization switching in cobalt layers ${ }^{3-6}$ is strong evidence that spin transfer dominates charge current-induced Oersted magnetic fields in mesoscopic small structures. Spin-transfer devices are promising for applications by the ability to excite and probe the dynamics of magnetic moments at small length scales. Reversing magnetizations with little power consumption can be utilized in current-controlled magnetic memory elements. As a result the spin-transfer effect has already been the subject of several theoretical studies. $^{7-15}$

The torque can be formulated by scattering theory in terms of the spin dependence of the reflection coefficients of the interface and the incoherence of spin-up and -down states inside the ferromagnet. This leads to a destructive interference of the component of the spin current perpendicular to the magnetization over the ferromagnetic decoherence length, which is smaller than the mean free path for not too weak ferromagnets. ${ }^{8-14}$ In this paper we solve the spindependent diffusion equation for a multilayer system consisting of two reservoirs, three normal metal layers, and two ferromagnetic layers (see Fig. 1), generalizing the approach of Valet and Fert ${ }^{16}$ to noncollinear systems.

Here we present an approach based on a diffusion equation that reveals the main physical effects of spin-flip scattering in different parts of the multilayer on the spin transfer: Spin-flip scattering in the middle normal metal N2 reduces the spin transfer, whereas spin-flip scattering in the outer normal metals N1 and N3 can enhance the spin transfer. Spin-flip at interfaces is not considered analytically, although in Appendix B we include it into our approach. Since interface spin-flip is again a smaller correction to most (but not all $^{17}$ ) interfaces, the present analytic results are therefore quite generally valid. We mainly focus on relatively large systems in which the bulk resistance dominates. Interfaces play an essential role in transferring the torques, but are assumed to not significantly increase the total as well as the mixing resistance (the inverse mixing conductance) in the limit of large systems. The inverse mixing conductance of a bulk (normal metal) layer with an interface is simply the sum of the inverse interface mixing conductance and the conventional bulk layer resistance. ${ }^{18}$ When the layer is sufficiently thick, the former can be disregarded. Physically this means that potential and spin-accumulation drops at the interfaces are so small that their contribution can be disregarded. A typical interface resistance for, e.g., $\mathrm{Co} / \mathrm{Cu}$, is $A R_{\text {surface }}$ $\sim \mathrm{f} \Omega \mathrm{m}^{2}$. The corresponding typical bulk resistance for clean and dirty $\mathrm{Co} / \mathrm{Cu}$ layer varies between $A R_{\text {bulk }}$ $=0.01 L[\mathrm{~nm}] \mathrm{f} \Omega \mathrm{m}^{2}$ and $0.1 L[\mathrm{~nm}] \mathrm{f} \Omega \mathrm{m}^{2}$ (see, for example, Refs. 19 and 20) where $L$ is the length of the layers expressed in $\mathrm{nm}$. In the presence of spin-flip the analytic expressions derived below are valid when the layers are thicker than $100 \mathrm{~nm}$ for pure samples and $10 \mathrm{~nm}$ for alloys, which is reasonable for experimental fabrication, and furthermore reveal qualitative effects of spin-flip relaxation processes on the spin torques for thinner layers. In the absence of spin-flip scattering our analytic results also hold for general structures (Appendix B). Related calculations of the torque and the magnetoresistance for submicron $\mathrm{Co} / \mathrm{Cu}$ multilayers using the Boltzmann equation were presented in Ref. 13.

The paper is organized as follows: in Sec. II we explain

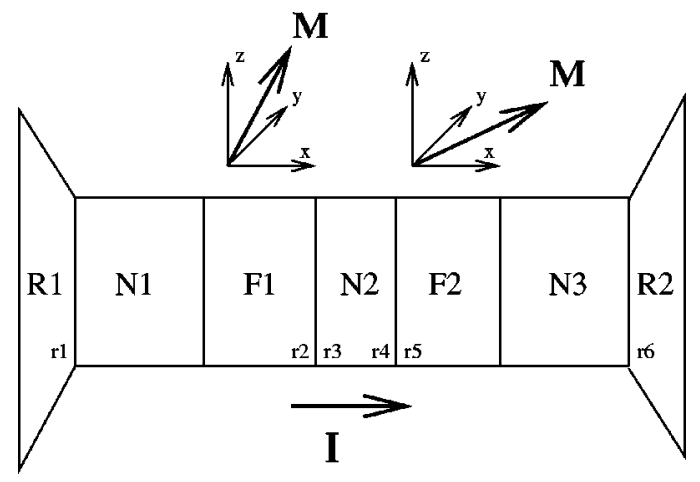

FIG. 1. N1-F1-N2-F2-N3 multilayer system with noncollinear magnetizations. 
the averaging mechanisms of spin transfer and the boundary conditions for the diffusion equation. The latter are formulated for a N1-F1-N2-F2-N3 multilayer system and solved analytically in the presence of spin-flip processes in the bulk layers in Secs. III and IV. In Sec. V we summarize our conclusion. In Appendix A, magnetoelectronic circuit theory ${ }^{8,10}$ is shown to be consistent with the results from the diffusion equation in the absence of spin-flip scattering. Interfaces are considered in Appendix B, where we also discuss the possibility to take into account spin-flip at interfaces.

\section{DIFFUSIVE APPROACH TO MULTILAYER SYSTEMS}

Electron states with spins that are not collinear to the magnetization direction are not eigenstates of a ferromagnet, but precess around the magnetization vector. In three dimensions, a noncollinear spin current is composed of many states with different Larmor frequencies which average out quickly in a ferromagnet as a function of penetration depth. The efficient relaxation of the nondiagonal terms in the spindensity matrix is equivalent to the suppression of spin accumulation noncollinear to the magnetization in the ferromagnet. ${ }^{8,10,13,14}$ This spin-dephasing mechanism does not exist in normal metals, in which the spin-wave functions remain coherent on the length scale of the spin-diffusion length, which can be of the order of microns. In ballistic systems, the spin transfer occurs over the ferromagnetic decoherence length $\lambda_{c}=1 /\left|k_{F}^{\uparrow}-k_{F}^{\downarrow}\right|$. In conventional ferromagnets the exchange energy is of the same order of magnitude as the Fermi energy, and $\lambda_{c}$ is of the order of the lattice constant. The strongly localized regime in which the mean free path is smaller than the inverse Fermi wave vector, $l$ $<1 / k_{F}$, is not relevant for elemental metals. In conventional metallic ferromagnets $l \gg 1 / k_{F}$, and the length scale of the spin transfer $\lambda_{c}$ is necessarily smaller than the mean free path $l$, and therefore is not affected by disorder (this argument does not hold for gradual interfaces and domain walls). The opposite limit was considered in Ref. 21 (although the authors intend to address the situation considered here in a forthcoming publication), where $\lambda_{c}=\sqrt{2 h D_{0} / J}\left(\lambda_{J}\right.$ in Ref. 21 ), or with $D_{0} \sim l^{2} / \tau, \lambda_{c} \sim l \sqrt{2 h / J \tau}$. The limit considered in Ref. 21 implies $2 h / J \tau>1$ or $\lambda_{c}>l$ and therefore does not hold for ferromagnetic conductors like $\mathrm{Fe}, \mathrm{Co}, \mathrm{Ni}$ and its alloys.

Semiclassical methods cannot describe processes on length scales smaller than the mean free path, and thus cannot properly describe abrupt interfaces. It is possible, however, to express boundary conditions in terms of transmission and reflection probabilities which connect the distribution functions on both sides of an interface, and have to be computed quantum mechanically. ${ }^{22}$ For transport, these boundary conditions translate into interface resistances, which arise from discontinuities in the electronic structure and disorder at the interface. This phenomenon was also extensively studied in the quasiclassical theory of superconductivity, ${ }^{23}$ where a generalized diffusion approach can be used in the bulk of the superconductor, provided that proper boundary conditions are employed at the interfaces.

Sharp boundaries can be taken into account by first- principles band-structure calculations. ${ }^{22}$ In collinear systems it is possible to circumvent the problem by replacing the interfaces by regions of a fictitious bulk material, the resistances of which can be fitted to experiments. This seems no longer possible when the magnetizations are noncollinear, because potential steps are essential for a description of the dephasing of the noncollinear spin current and the torque. However, in the case of a small imaginary part of the mixing interface conductance (which holds for intermetallic interfaces) this again becomes the correct procedure, as shown in Appendix B.

We wish to model the multilayer system (Fig. 1) by the diffusion equation and interface boundary conditions. Let $\hat{f}(\varepsilon)$ be the $2 \times 2$ distribution matrix at a given energy $\varepsilon$ and $\hat{I}$ the $2 \times 2$ current matrix in spin space. It is convenient to expand these matrices into scalar particle and vector spin contributions $\hat{f}=\hat{1} f_{0}+\hat{\sigma} \cdot \mathbf{f}_{s}, \hat{I}=\left(\hat{1} I_{0}+\hat{\sigma} \cdot \mathbf{I}_{s}\right) / 2$. For normal metals $\hat{f}^{N}=\hat{1} f_{0}^{N}+\hat{\sigma} \cdot \mathbf{s}$, where $f_{0}^{N}$ is the local charge-related chemical potential and the spin distribution function has magnitude $f_{s}^{N}$ and direction s. In the ferromagnet $\hat{f}^{F}=\hat{1} f_{0}^{F}$ $+\hat{\sigma} \cdot \mathbf{m} f_{s}^{F}=\hat{1}\left(f_{\uparrow}+f_{\downarrow}\right) / 2+\hat{\sigma} \cdot \mathbf{m}\left(f_{\uparrow}-f_{\downarrow}\right) / 2$, where $f_{\uparrow}$ and $f_{\downarrow}$ are the diagonal elements of the distribution matrix when the spin-quantization axis is parallel to the magnetization in the ferromagnet $\mathbf{m}$.

The diffusion equation describes transport in both the normal metal and the ferromagnet. We first consider a single interface and disregard spin-flip scattering. The particle and spin currents in the normal metal with diffusion constant $D$ are $j=D \partial_{x} f_{0}^{N}$ and $\mathbf{j}_{s}^{N}=D \partial_{x} \mathbf{f}_{s}^{N}$, respectively. The particle and spin currents are then conserved:

$$
D \partial_{x}^{2} f_{0}^{N}=0, \quad D \frac{\partial^{2}}{\partial x^{2}} \mathbf{f}_{s}^{N}=0 .
$$

In the ferromagnet the particle and spin currents are $j$ $=D_{\uparrow} \partial_{x} f_{\uparrow}+D_{\downarrow} \partial_{x} f_{\downarrow}$ and $\mathbf{j}_{s}^{F}=\mathbf{m} \partial_{x}\left(D_{\uparrow} f_{\uparrow}-D_{\downarrow} f_{\downarrow}\right)$ [see Ref. 10, Eqs. (38)-(39)], where $D_{\uparrow}$ and $D_{\downarrow}$ are the diffusion constants for spin-up and -down electrons. Current conservation of the spin components parallel and antiparallel to the magnetization direction in the ferromagnet read

$$
D_{\uparrow} \partial_{x}^{2} f_{\uparrow}=0, \quad D_{\downarrow} \partial_{x}^{2} f_{\downarrow}=0 .
$$

Equations (1) and (2) are applicable only inside the bulk layers. The boundary conditions at the interface arise from the continuity of the particle and spin distribution functions on the normal and the ferromagnetic metal sides ${ }^{8,10}$ :

$$
\begin{gathered}
\left.f_{s}^{N}\right|_{\mathrm{N} \text {-surface }}=\left(f_{\uparrow}+f_{\downarrow}\right) /\left.2\right|_{\mathrm{F} \text {-surface }}, \\
\left.\mathbf{f}_{s}^{\mathrm{N}}\right|_{\mathrm{N} \text {-surface }}=\mathbf{m}\left(f_{\uparrow}-f_{\downarrow}\right) /\left.2\right|_{\text {F-surface }} .
\end{gathered}
$$

Furthermore, particle current is conserved ${ }^{8,10}$ :

$$
\left.\left[D \partial_{x} f_{0}^{N}\right]\right|_{\mathrm{N} \text {-surface }}=\left.\partial_{x}\left(D_{\uparrow} f_{\uparrow}+D_{\downarrow} f_{\downarrow}\right)\right|_{\text {F-surface }} \text {. }
$$

We have discussed above why the noncollinear component of the spin-accumulation decays on the length scale of the order of the lattice spacing. This leads to the third boundary 
condition at the F-N interface, namely, that the spin current is conserved only for the spin component parallel to the magnetization direction, ${ }^{8,10}$

$$
\left.\left[D \partial_{x} \mathbf{f}_{s}^{N}\right]\right|_{\mathrm{N} \text {-surface }}=\left.\mathbf{m} \partial_{x}\left(D_{\uparrow} f_{\uparrow}-D_{\downarrow} f_{\downarrow}\right)\right|_{\text {F-surface }}+\vec{\tau}
$$

where $\vec{\tau} \perp \mathbf{m}$ is the nonconserved part of the spin current leading to torques acting on the magnetization in the ferromagnet.

Solving these equations, we recover Eq. (A3) with a mixing conductance as found by the magnetoelectric circuit theory (Appendix A). ${ }^{8,10}$ The magnetoelectronic circuit theory is thus equivalent to the diffusion approach when the system size is larger than the mean free path. Note that the boundary conditions above do not contain explicit reference to interface conductance parameters and are therefore valid only when bulk resistances are sufficiently larger than the interface resistances. The advantage of using the diffusion equation is that we can now easily derive simple analytical results, also in the presence of spin-flip relaxation. In normal as well as ferromagnetic metals, spin-flip scattering leads to

$$
\partial_{x} j_{0}=0, \frac{\partial}{\partial x} \mathbf{j}_{s}=\mathbf{f}_{s} / \tau_{s f}
$$

where the spin-flip relaxation time $\tau_{s f}$ is a material dependent parameter.

\section{RESULTS FOR SYSTEMS WITHOUT SPIN-FLIP}

Let us now apply this method to the spin transfer in a N1-F1-N2-F2-N3 system (Fig. 1) to obtain explicit results for the figure of merit, viz. the ratio of the spin torque to the charge current through (or voltage bias across) the system.
The layers are characterized by the lengths $L_{N 1}, L_{F 1}, L_{N 2}$, $L_{F 2}$, and $L_{N 3}$ and by diffusion constants $D_{N 1}, D_{F 1, \uparrow(\downarrow)}$, $D_{N 2}, D_{F 2, \uparrow(\downarrow)}$, and $D_{N 3}$ for each normal and ferromagnetic metal layer, respectively. The resistances of the system are $R_{N 1}, R_{F 1, \uparrow}, R_{F 1, \downarrow}, R_{N 2}, R_{F 2, \uparrow}, R_{F 2, \downarrow}$, and $R_{N 3}$ with, for example, $R_{N 1}=L_{N 1} /\left(A_{N 1} D_{N 1}\right)$ and $R_{F 1, \uparrow}=L_{F 1} /\left(A_{F 1} D_{F 1, \uparrow}\right)$ ( $L$ and $A$ are the length and cross section of a layer respectively). Let us initially disregard spin-flip scattering.

The continuity of the spin-current at the interface N1-F1 can easily be shown from Eqs. (1), (4), and (6). As a result the two layers N1-F1 behave effectively like a single ferromagnetic layer with renormalized resistances:

$$
\begin{aligned}
& \widetilde{R}_{F 1, \uparrow}=R_{F 1, \uparrow}+2 R_{N 1}, \\
& \widetilde{R}_{F 1, \downarrow}=R_{F 1, \downarrow}+2 R_{N 1} .
\end{aligned}
$$

The same is true for the interface F2-N3. As a result it is sufficient to treat only the F1-N-F2 system. In general, there are spin-current discontinuities at the interfaces F1-N and $\mathrm{N}-\mathrm{F} 2$ which, due to momentum conservation, lead to torques acting on the magnetic moments in the ferromagnetic layers. Taking into account all diffusion equations (1) and (2) and boundary conditions (3)-(6), and also introducing the parameters $R=R_{N 2}, R_{i \pm}=\left(\widetilde{R}_{F i, \uparrow} \pm \widetilde{R}_{F i, \downarrow}\right) / 4$, where $i=1,2$, the torques can be written as

$$
\vec{\tau}_{1}=I_{0} R_{2-} \frac{\left(R+R_{1+}-\alpha R_{1-} R_{2+} / R_{2-}\right)}{\left(R+R_{2+}\right)\left(R+R_{1+}\right)-\alpha^{2} R_{1+} R_{2+}}\left(\alpha \mathbf{m}_{1}-\mathbf{m}_{2}\right)
$$

$$
\vec{\tau}_{2}=I_{0} R_{1-} \frac{R+R_{2+}-\alpha R_{2-} R_{1+} / R_{1-}}{\left(R+R_{2+}\right)\left(R+R_{1+}\right)-\alpha^{2} R_{1+} R_{2+}}\left(\mathbf{m}_{1}-\alpha \mathbf{m}_{2}\right),
$$

where $\tau_{1}$ and $\tau_{2}$ are torques acting on the magnetizations of the first and second ferromagnet respectively, $\alpha=\left(\mathbf{m}_{1} \cdot \mathbf{m}_{2}\right)$ $=\cos \theta, \theta$ being the angle between the magnetizations. The resistance becomes

$$
\Re(\theta)=R+R_{1+}+R_{2+}-\frac{R_{1-}^{2}+2 \alpha R_{1-} R_{2-}+R_{2-}^{2}+\left(1-\alpha^{2}\right)\left(R_{1-}^{2} R_{2+}+R_{2-}^{2} R_{1+}\right) / R}{R+R_{1+}+R_{2+}+R_{1+} R_{2+}\left(1-\alpha^{2}\right) / R} .
$$

It is worthwhile to rewrite Eq. (9) using the effective polarization $P=R_{-} / R_{+}$(which is the polarization of a current flowing through $\mathrm{F}$ or N-F layers connected to reservoirs) and the ferromagnet charge current resistance $R_{i}=R_{i+}$. The (absolute values of the) torques are then

$$
\left|\tau_{1}\right|=\frac{\left|1+R / R_{1}-\alpha P_{1} / P_{2}\right|}{\left(1+R / R_{2}\right)\left(1+R / R_{1}\right)-\alpha^{2}} I_{0} P_{2}|\sin \theta|
$$

$$
\left|\tau_{2}\right|=\frac{\left|1+R / R_{2}-\alpha P_{2} / P_{1}\right|}{\left(1+R / R_{1}\right)\left(1+R / R_{2}\right)-\alpha^{2}} I_{0} P_{1}|\sin \theta|
$$

As one can see from Eqs. (9) there is an asymmetry with respect to current inversion. For example, if only one polarization can rotate (one ferromagnet is much wider than the other or exchange biased), domains in the two magnetic layers can be aligned antiparallel by currents flowing in one direction, and reoriented parallel by reversing the current 


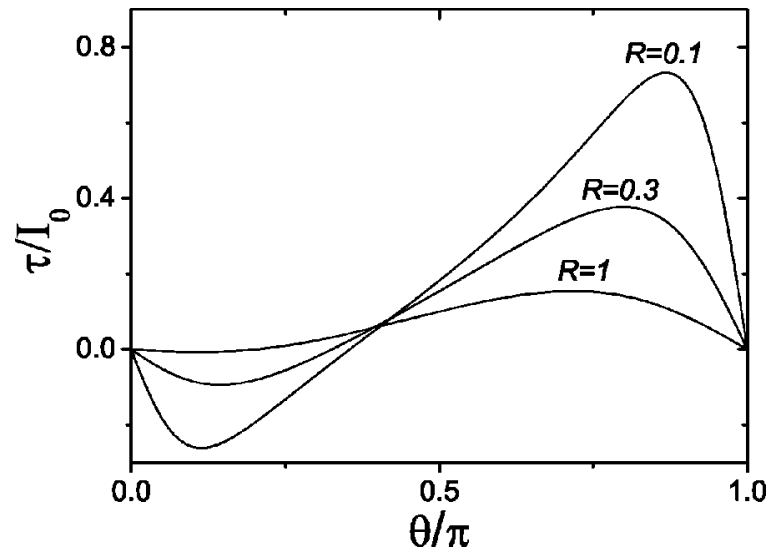

FIG. 2. Torque acting on the first ferromagnet as a function of the relative angle between the two magnetizations for different normal metal resistances (the resistances are expressed in units $R_{1}$ $=R_{2}, P_{1}=0.5$, and $P_{2}=0.2$ ).

flow. This happens because only one state (parallel or antiparallel) is at equilibrium for a fixed direction of the current. If the currents are large enough (depending on other sources of torques such as external fields, magnetocrystalline anisotropy and damping) the magnetization will flip, which can be monitored by a change in the total resistance of

$$
\frac{R(\uparrow \downarrow)-R(\uparrow \uparrow)}{R(\uparrow \downarrow)}=\frac{4 R_{1-} R_{2-}}{R^{2}+\left(R_{1+}+R_{2+}\right)^{2}-\left(R_{1-}+R_{2-}\right)^{2}} .
$$

In the case of unit polarization and $R \approx 0$ the relative resistance change [Eq. (12)] can be $100 \%$. This asymmetry was predicted by spin-transfer theory, ${ }^{1}$ and was observed experimentally. ${ }^{3-5}$ Note, however, that in these experiments the mean free path is comparable to the size of the systems, and the present theory cannot be directly applied.

From Eq. (11) follows that the torques are equal to zero for parallel and antiparallel alignments. When the numerator of Eq. (11) $1+R / R_{1(2)}-\alpha P_{1(2)} / P_{2(1)}$ never vanishes, the torque increases with $\theta$ from zero to a maximal value which corresponds to an angle larger than $\pi / 2$ and vanishes again when configurations become antiparallel. When the nominator of Eq. (9) does vanish for some angle $\theta_{0}$, the absolute value of torque has a local maximum before $\theta_{0}$ (see Fig. 2). In principle, it is possible to have an equilibrium magnetization angle $\theta=\theta_{0}$ for one current direction, whereas the magnetization angle $\theta=0$ or $\pi$ for the opposite current direction (this can lead to asymmetry for the transition from the antialigned state to the aligned state in comparison with the transition from aligned to antialigned state, as observed experimentally ${ }^{6}$ ).

We propose a setup in which only one magnetization can rotate (usually it is achieved by taking one ferromagnetic layer much wider than the other or by exchange biasing). If one ferromagnetic layer (for example the first one) has a resistance $R_{1} \ll R$ and the other $R_{2}>R$, the torque $\tau_{2}$ vanishes whereas the other torque can be simplified to

$$
\left|\tau_{1}\right|=I_{0} P_{2}|\sin \theta| \text {. }
$$

The maximal torque in this setup occurs when the magnetizations of ferromagnet F1 and ferromagnet F2 are perpendicular.

In general, the spin torque is maximal when the resistance $R$ of the normal metal vanishes, as could have been expected since this also gives the maximum magnetoresistance effect. In Eqs. (9) and (11) the size of the magnets does not play a dominant role for small normal metal resistances. In this case the torques depend mainly on the polarizations.

\section{RESULTS FOR SYSTEMS WITH SPIN-FLIP}

So far, we have disregarded spin-flip scattering, which can be included readily, however. Here the system N1-F1-N2F2-N3 is analyzed, and spin-flip in each normal metal part is considered separately. Introducing spin-flip in N1 and N3 leads to a simple result: Eq. (8) without spin-flip remains valid, but with modified spin-flip resistances,

$$
R_{N 1(N 3)}^{s f}=R_{N 1(N 3)} \frac{\tanh \left(L_{N 1(N 3)} / l_{s d}\right)}{\left(L_{N 1(N 3)} / l_{s d}\right)},
$$

where $l_{s d}$ is the normal metal spin-flip diffusion length. When $L \gg l_{s d}$, the resistance is governed by the spin-flip diffusion length $l_{s d}$, which means that only part of the metal takes part in the spin transfer whereas the rest plays the role of the reservoir. This reduction of the active thickness of the device can lead to an effective polarization increase by decreasing the effect of $R_{N}$ in Eq. (8). Spin-flips in the middle normal metal have a larger impact. The torques in the presence of spin-flips in N2 read

$$
\begin{aligned}
& \left|\tau_{1}\right|=\frac{\beta+R^{s f} / R_{1}-\alpha P_{1} / P_{2}}{\left(\beta+R^{s f} / R_{2}\right)\left(\beta+R^{s f} / R_{1}\right)-\alpha^{2}} I_{0} P_{2}|\sin \theta|, \\
& \left|\tau_{2}\right|=\frac{\beta+R^{s f} / R_{2}-\alpha P_{2} / P_{1}}{\left(\beta+R^{s f} / R_{1}\right)\left(\beta+R^{s f} / R_{2}\right)-\alpha^{2}} I_{0} P_{1}|\sin \theta|,
\end{aligned}
$$

where $\beta=\cosh \left(L / l_{s d}\right)$ and $P_{1(2)}$ and $R_{1(2)}$ are given by Eqs. (8) and (14). $R^{s f}$ is an effective normal metal resistance:

$$
R^{s f}=R \frac{\sinh \left(L / l_{s d}\right)}{L / l_{s d}}
$$

For $L \geqslant l_{s d}$ the torque is significantly reduced by spin-flips, becoming exponentially small for longer samples.

Let us now consider spin-flips in the ferromagnet. The treatment of the N1-F1-N2-F2-N3 system is cumbersome, so let us concentrate on the simple case of an F-N-F system. In that case formulas remain unchanged, provided $R_{+}$and $R_{-}$ are renormalized as

$$
R_{1(2)-}^{s f}=R_{1(2)-} \frac{\tanh \left(L_{F 1(F 2)} / l_{s d}^{F}\right)}{L_{F 1(F 2)} / l_{s d}^{F}},
$$




$$
R_{1(2)+}^{s f}=R_{1(2)+} \frac{\tanh \left(L_{F 1(F 2)} / l_{s d}^{F}\right)}{L_{F 1(F 2)} / l_{s d}^{F}} .
$$

where $l_{s d}^{F}$ is the ferromagnet spin-flip diffusion length. These resistances should be used in Eqs. (9) for the torques in F-N-F systems. If spin-flip in the normal metal exists, then formulas (15) should be used. Equations (17) imply that there is no polarization change [as defined below Eqs. (9)] and only the ferromagnet resistances $R_{1(2)}$ are affected. For $L \gg l_{s d}^{F}$ the bulk of the ferromagnet behaves like a reservoir (just like the normal metal in the same limit) and only a slice with thickness $l_{s d}^{F}$ is active. In general, spin-flip in the ferromagnet leads to reduced torques as $R_{1(2)}$ becomes smaller. The effect may be quite small as long as the resistance of the ferromagnet is sufficiently larger than that of the normal metal [this can also be seen from Eqs. (8) and (11)], so that the polarization of the current is maintained.

Finally we would like to discuss magnetoresistance and torque for the symmetric $R_{1+}=R_{2+}$ and $R_{1-}=R_{2-}$. For the angular magnetoresistance we extract from (10) the formula observed by Pratt ${ }^{24}$ and also shown to be universal for any disordered F-N-F perpendicular spin valves in Ref. 18,

$$
\frac{R(\theta)-R(0)}{R(\pi)-R(0)}=\frac{1-\cos \theta}{\chi(1+\cos \theta)+2},
$$

with one parameter $\chi$ that is given by circuit theory,

$$
\chi=\frac{1}{1-p^{2}} \frac{|\eta|^{2}}{\operatorname{Re\eta }}-1,
$$

in terms of the normalized mixing conductance $\eta$ $=2 g_{\uparrow \downarrow} / g$, the polarization $p=\left(g_{\uparrow}-g_{\downarrow}\right) / g$, and the average conductance $g=g_{\uparrow}+g_{\downarrow}$. As we do not take interface resistances into account, in our case the parameters can be expressed only via bulk resistances: $g=1 /\left(R_{\uparrow}+R\right)+1 /\left(R_{\downarrow}\right.$ $+R), \eta=2 /(R g), p=2 R_{-} /\left(2 R_{+}+R\right)$. From Eqs. (11) and (10) the analytical expressions of the spin torque on either ferromagnet for current and voltage biased systems read

$$
\begin{gathered}
|\tau|=\frac{p(\chi+1)|\sin \theta|}{\chi(\cos \theta+1)+2} I_{0}, \\
|\tau|=\frac{p g}{2} \frac{\eta|\sin \theta|}{(\eta-1) \cos \theta+1+\eta} \frac{\left|\mu_{l}-\mu_{r}\right|}{2 \pi},
\end{gathered}
$$

where $\mu_{l(r)}$ is the chemical potential in the left (right) ferromagnet. In the presence of spin-flip for the angular magnetoresistance we can write (restricting ourself to F-N-F case again)

$$
\frac{R(\theta)-R(0)}{R(\pi)-R(0)}=\frac{[1+\chi(\beta-1) / 2](1-\cos \theta)}{\chi(\beta+\cos \theta)+2},
$$

where all parameters should be calculated according to Eqs. (16) and (17). The dependences of the torque on angle now read

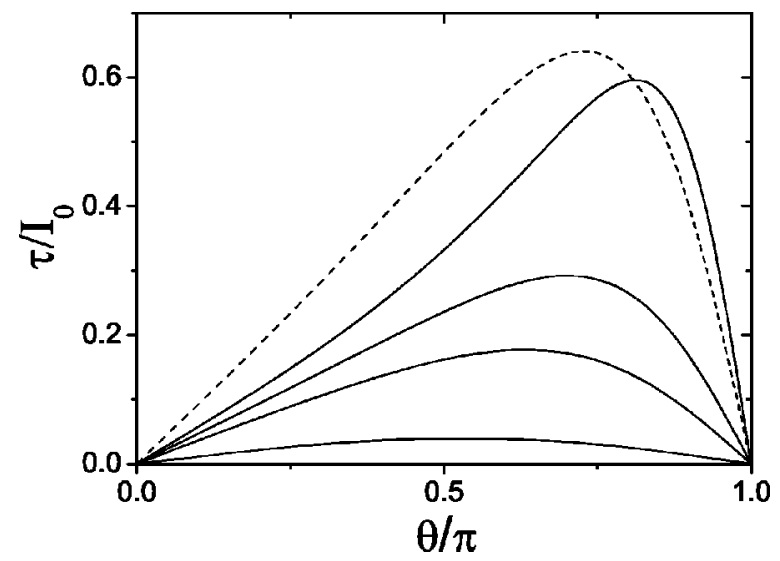

FIG. 3. Torque on each ferromagnet as a function of the relative angle between the two magnetizations for different spin-flip diffusion lengths in the normal metal (the resistance $R=0.2$ is expressed in units $R_{1}=R_{2}, P_{1}=P_{2}=0.4$, and $L / l_{s d}=0,1,1.5$, and 3 , and the lower plot corresponds to the higher ratio). With the dashed line we plot Slonczewski's result (Ref. 1) for the same polarization.

$$
\begin{gathered}
|\tau|=\frac{p(\chi+1)|\sin \theta|}{[\chi(\cos \theta+\beta)+2]} I_{0}, \\
|\tau|=\frac{p g}{2} \frac{\eta|\sin \theta|}{A_{1} \cos \theta+A_{2}} \frac{\left|\mu_{l}-\mu_{r}\right|}{2 \pi},
\end{gathered}
$$

where we introduced four parameters

$$
\begin{aligned}
& A_{1}=-\frac{p^{2}}{1+\frac{\chi}{2} \frac{\beta-1}{\chi+1}}\left(1+\frac{\chi \beta+\chi}{2}\right) \\
& +\chi\left(1+\frac{\left(\kappa_{1}+\chi \kappa_{2}\right)}{\chi+1}-\kappa_{2} p\right), \\
& A_{2}=\frac{p^{2}}{1+\frac{\chi}{2} \frac{\beta-1}{\chi+1}}\left(1-\frac{\chi \beta+2}{2}(\beta+1)\right) \\
& +\left(1+\frac{\left(\kappa_{1}+\chi \kappa_{2}\right)}{\chi+1}-\kappa_{2} p\right)(\chi \beta+2),
\end{aligned}
$$

and

$$
\kappa_{1}=\frac{L_{N} / l_{N, s d}}{\sinh \left(L_{N} / l_{N, s d}\right)}-1, \quad \kappa_{2}=\frac{L_{F} / l_{F, s d}}{\tanh \left(L_{F} / l_{F, s d}\right)}-1 .
$$

An interesting result can be drawn from Eqs. (22) and (23) by comparison with the Eqs. (18) and (20). In order to fit the torque and the magnetoresistance in the presence of spin-flip we need an additional parameter $\beta$ [defined in Eqs. (15)], which depends only on the spin-flip diffusion length in the normal metal spacer. The general form of Eqs. (22) and (23) with only two parameters seems to be valid even in the presence of interfaces, but this has to be confirmed by future studies. Equation (24) is cumbersome, depending explicitly on the diffusion length in the ferromagnets. In Fig. 3 we plot results of Eq. (23) for different spin-diffusion lengths in the 
normal metal. The smaller diffusion length corresponds to smaller torques. The curves only qualitatively resemble Slonczewski's result for ballistic systems, but it should be pointed out that for the limit $p=1$ and $\eta=2$, both approaches result in the same formula.

\section{CONCLUSION}

We investigated transport in multilayer systems in the diffusive limit with arbitrary magnetizations in the ferromagnetic layers. The boundary conditions for diffusion equations including spin transfer were discussed, and analytic expressions for the magnetization torques and the angular magnetoresistance were obtained. The torque can be engineered not only via the geometry of the samples (such as the layer thicknesses), but also via the materials, the ferromagnetic polarization being an important parameter. The asymmetry with respect to the current flow direction has been addressed and the resistance change under magnetization reversal was calculated for different current directions. The effect of spin-flip in the normal metal and ferromagnet was studied analytically. Spin-flip in the center normal metal suppresses the spin transfer, whereas spin-flip in the outer normal metals can effectively increase the polarization and spin transfer. The spin-flip processes in the ferromagnet also diminish the spin transfer, but not as drastically as long as the resistance of the ferromagnet is larger than the normal metal resistance. Finally we show in Appendix A that the diffusive approach with carefully chosen boundary conditions leads to results which coincide with those from circuit theory.

\section{ACKNOWLEDGMENTS}

We are grateful to Yuli Nazarov, Yaroslav Tserkovnyak, and Daniel Huertas-Hernando for stimulating discussions. This work was supported in part by the NEDO International Joint Research Grant Program "Nano-magnetoelectronics," NSF Grant No. DMR 99-81283 and DARPA Award No. MDA 972-01-1-0024.

\section{APPENDIX A: CIRCUIT THEORY APPROACH TO DIFFUSIVE SYSTEMS}

Here we show that the diffusion approach is equivalent to circuit theory and that the mixing conductance is also a valid concept in systems which are dominated by bulk transport. ${ }^{25}$ We consider an F1-N2-F2 system Fig. 1 (N1 and N3 can also be included) connected to two reservoirs R1 and R2 with negligible interface resistances. Note that this does not mean that the interface is neglected, because it plays an essential role in the boundary conditions as mentioned in the main text. Since the system is diffusive, a thin slice of a ferromagnet or a normal metal can be considered as a node. The mixing conductance can be written in terms of the reflection and transmission coefficients, and incorporates any kind of contacts, e.g., tunnel, diffusive, and ballistic contacts. We are free to define interface resistors via the location of the nodes. Here it is chosen such that the interface width is larger than the ferromagnetic decoherence length but smaller than the mean free path. We introduce six nodes: $\mathrm{r} 1$ is in $\mathrm{R} 1$ just before the interface, $\mathrm{r} 2$ and $\mathrm{r} 3$ are before and after the F1-N2 interface, $\mathrm{r} 4$ and $\mathrm{r} 5$ are before and after the N2-F3 interface, and $\mathrm{r} 6$ is in R2 just after the interface (Fig. 1).

Let us first find the charge and the spin current in F1 (F2) at the interface where the spin transfer takes place. The currents read

$$
\begin{gathered}
I_{0}=\left(G^{\downarrow}+G^{\uparrow}\right)\left(f_{0}^{F}-f_{0}^{N}\right)+\left(G^{\downarrow}-G^{\uparrow}\right) f_{s}^{F}, \\
\mathbf{I}_{s}=\mathbf{m}\left[\left(G^{\downarrow}-G^{\uparrow}\right)\left(f_{0}^{F}-f_{0}^{N}\right)+\left(G^{\downarrow}+G^{\uparrow}\right) f_{s}^{F}\right],
\end{gathered}
$$

where $f_{0}^{N}$ is the particle distribution function in $\mathrm{r} 1$ (r6) and $f_{0}^{F}$ is the spin distribution function in $\mathrm{r} 2(\mathrm{r} 5)$. The distribution function at $\mathrm{r} 2$ and $\mathrm{r} 3$ ( $\mathrm{r} 4$ and $\mathrm{r} 5$ ) is identical due to the continuity boundary condition. The spin-current between the ferromagnet reservoir $\mathrm{r} 2(\mathrm{r} 5)$ and the normal metal reservoir $\mathrm{r} 4$ (r3) driven by the nonequilibrium distributions can be found by using circuit theory,

$$
\begin{aligned}
\mathbf{I}_{1(2)}= & \mathbf{m} 2 G_{N}\left(f_{s}^{F}-\mathbf{s} \cdot \mathbf{m} f_{s}^{N}\right)-2 \operatorname{Re} G^{\uparrow \downarrow} f_{s}^{N}[\mathbf{s}-(\mathbf{s} \cdot \mathbf{m}) \mathbf{m}] \\
& +(\mathbf{s} \times \mathbf{m}) 2 \operatorname{Im} G^{\uparrow} \downarrow f_{s}^{N},
\end{aligned}
$$

where the spin accumulation in the normal metal reservoir $\mathrm{r} 4$ $(\mathrm{r} 3)$ is given by the unit vector $\mathbf{s}$ and the spin distribution function $f_{s}^{N}$. Use was made of $G^{\downarrow}=G^{\uparrow}=G_{N}$ because $\mathrm{r} 2(\mathrm{r} 5)$ is close to the interface. The component of the current perpendicular to the magnetization $\mathbf{m}$ is transferred to the magnetization at the interface whereas the parallel component is conserved. The torque acting on the magnetization in F1 (F2) therefore becomes

$$
\begin{aligned}
\vec{\tau}_{1(2)}= & -2 \operatorname{Re} G^{\uparrow \downarrow} f_{s}^{N}\left[\mathbf{s}^{N}-\left(\mathbf{s}^{N} \cdot \mathbf{m}\right) \mathbf{m}\right] \\
& +\left(\mathbf{s}^{N} \times \mathbf{m}\right) 2 \operatorname{Im} G^{\uparrow \downarrow} f_{s}^{N} .
\end{aligned}
$$

The mixing conductance is related to the reflection coefficients of an electron from the normal metal to the ferromagnet:

$$
G_{\uparrow \downarrow}=\sum_{n m}\left[\delta_{n m}-\left(r_{n m}^{\uparrow}\right) * r_{n m}^{\downarrow}\right] .
$$

Let us now evaluate the mixing conductance for a disordered system. We assume that the junction consists of two connected parts. The normal metal section is described by a single scattering matrix for both spin- $\uparrow$ and spin- $\downarrow$ electrons. The ferromagnetic section requires two independent scattering matrices, one for spin- $\uparrow$ and one for spin- $\downarrow$ electrons. Scattering at the F-N boundary is disregarded here since it is assumed that the total resistance is dominated by the diffuse normal metal and ferromagnetic metal parts of the junction. The total reflection matrix $r^{\alpha}$ for spin- $\alpha$ electrons can then be found by concatenating the normal metal and ferromagnetic parts as

$$
r^{\alpha}=r_{N}+t_{N}^{\prime} r_{F}^{\alpha} \sum_{n=0}^{\infty}\left(r_{N}^{\prime} r_{F}^{\alpha}\right)^{n} t_{N} \equiv r_{N}+\chi^{\alpha}
$$


By inserting Eq. (A5) into the definition for the mixing conductance we find that it can be expressed as $G_{\uparrow \downarrow}=G_{N}$ $+\delta G_{\uparrow \downarrow}$, where

$$
\delta G_{\uparrow \downarrow}=\sum_{n m}\left[\left(r_{N}\right)_{n m}^{*} \chi_{n m}^{\downarrow}\right]+\left[\chi_{n m}^{\uparrow}\left(r_{N}\right)_{n m}\right]^{*}+\left(\chi_{n m}^{\uparrow}\right)^{*} \chi_{n m}^{\downarrow} .
$$

Equation (A6) depends on the phase difference between the scattering paths of spin-up and -down electrons. It is assumed that there are no correlations between the scattering matrices of the spin- $\uparrow$ and spin- $\downarrow$ electrons in the ferromagnetic part, which is consistent with the small coherence length. Consequently, in a diffusive systems $\delta G_{\uparrow \downarrow}=0$. However, the up- and down-spin parts of the total scattering matrix of the combined normal metal and ferromagnetic system are correlated since both spin directions see the same scattering centers in the normal metal part. This leads to the conclusion that, for a diffusive hybrid system,

$$
G_{\uparrow \downarrow}^{D}=G_{N} \cdot
$$

From Eqs. (A1)-(A3) and taking into account Eq. (A7), and noting that $2 G_{N}=1 / R$ and $G_{\downarrow}=1 / R_{\downarrow}, G_{\uparrow}=1 / R_{\uparrow}$ one can easily find Eq. (11).

\section{APPENDIX B: TREATMENT OF INTERFACES}

We show in this Appendix how interfaces can be included into our approach. The general form of the boundary conditions at an $\mathrm{F} \mid \mathrm{N}$ interface can be written

$$
\begin{gathered}
\left.f_{s}^{N}\right|_{N-\text { interface }}=\left.\left(f_{\uparrow}+f_{\downarrow}\right)\right|_{F-\text { interface }}+\Delta f, \\
\left.\mathbf{f}_{s}^{N}\right|_{N-\text { interface }}=\left.\mathbf{m}\left(f_{\uparrow}+f_{\downarrow}\right)\right|_{F-\text { interface }}+\Delta \mathbf{f}_{s} .
\end{gathered}
$$

In the absence of spin-flip scattering at the interface the potential $\Delta f$ and spin-accumulation $\Delta \mathbf{f}_{s}$ drops can be found from the magnetoelectronic circuit theory as follows:

$$
\begin{gathered}
I_{0}=\left(G^{\uparrow}+G^{\downarrow}\right) \Delta f+\left(G^{\uparrow}-G^{\downarrow}\right)\left(\Delta \mathbf{f}_{s} \cdot \mathbf{m}\right), \\
\mathbf{I}_{s}=\mathbf{m}\left[\left(G^{\uparrow}-G^{\downarrow}\right) \Delta f+\left(G^{\uparrow}+G^{\downarrow}\right)\left(\Delta \mathbf{f}_{s} \cdot \mathbf{m}\right)\right]+2 \operatorname{Re} G^{\uparrow \downarrow} \mathbf{f}_{s}^{N} \\
+2 \operatorname{Im} G^{\uparrow \downarrow}\left(\mathbf{f}_{s}^{N} \times \mathbf{m}\right) .
\end{gathered}
$$

When the imaginary part of the mixing conductance is small, the interface can be replaced by a fictitious bulk material. Suppose we add a slice of ferromagnet as well as a slice of normal metal with parameters $R_{ \pm}=\left(R_{\uparrow} \pm R_{\downarrow}\right) / 4$ and $R_{N}$ respectively, the potential and spin-accumulation drops read

$$
\begin{gathered}
\Delta f=I R_{N}+\left(\mathbf{m} \cdot \mathbf{I}_{s}\right) R_{-}+I_{0} R_{+}, \\
\Delta \mathbf{f}_{s}=\mathbf{I}_{s} R_{N}+\mathbf{m}\left(\mathbf{m} \cdot \mathbf{I}_{s}\right) R_{+}+\mathbf{m} I_{0} R_{-} .
\end{gathered}
$$

In general, Eqs. (B5) and (B6) have some $R_{N}$ and $R_{ \pm}$as a solution.

Taking into account results from the Appendix A and Eqs. (B3)-(B6), one can immediately derive that the ficticious bulk layers should obey

$$
R_{N}=1 / G^{\uparrow \downarrow}, \quad R_{\uparrow}=1 / G_{\uparrow}-1 / G^{\uparrow \downarrow}, \quad R_{\downarrow}=1 / G_{\downarrow}-1 / G^{\uparrow \downarrow} .
$$

When interface and bulk spin-flip may be disregarded and $\operatorname{Im} G^{\uparrow \downarrow}=0$, the interface thus leads to the following renormalized up, down and normal metal resistances of adjacent layers $\left(R_{N}^{f}, R_{\uparrow}^{f}\right.$, and $\left.R_{\downarrow}^{f}\right)$ :

$$
\begin{gathered}
R_{N}^{f}=R_{N}+1 / G^{\uparrow \downarrow}, \quad R_{\uparrow}^{f}=R_{\uparrow}+1 / G_{\uparrow}-1 / G^{\uparrow \downarrow}, \\
R_{\downarrow}^{f}=R_{\downarrow}+1 / G_{\downarrow}-1 / G^{\uparrow \downarrow} .
\end{gathered}
$$

Recent experiments indicate that interface spin-flip scattering may not be neglected in many systems. ${ }^{26}$ It is straightforward to introduce spin-flip diffusion into the ficticious layers, although difficulties arise from the necessity to link the analytical solutions between regions with different spinflip rates, $l / l_{s d}$. Only when the $\mathrm{N}(\mathrm{F})$ fictitious layer has the same spin-flip rate as the adjacent $\mathrm{N}(\mathrm{F})$ layer can we carry out analytic calculations. In this case the total system is equivalent to a bulk system with resistances given by Eqs. (B8) [these resistances should be used in Eqs. (14), (16), and (17)]. Otherwise, the problem becomes too cumbersome for an analytic treatment and has to be studied numerically.

R3213 (1998).

${ }^{8}$ A. Brataas, Y. V. Nazarov, and G. E. W. Bauer, Phys. Rev. Lett. 84, 2481 (2000).

${ }^{9}$ X. Waintal, E. B. Myers, P. W. Brouwer, and D. C. Ralph, Phys. Rev. B 62, 12317 (2000).

${ }^{10}$ A. Brataas, Y. V. Nazarov, and G. E. W. Bauer, Eur. Phys. J. B 22, 99 (2001).

${ }^{11}$ K. Xia, P. J. Kelly, G. E. W. Bauer, I. Turek, J. Kudrnovsky, and V. Drchal, Phys. Rev. B 63, 064407 (2001).

${ }^{12}$ K. Xia, P. J. Kelly, G. E. W. Bauer, A. Brataas, and I. Turek, Phys. Rev. B 65, 220401(R) (2002).

${ }^{13}$ M. D. Stiles and A. Zangwill, J. Appl. Phys. 91, 6812 (2002).

${ }^{14}$ M. D. Stiles and A. Zangwill, Phys. Rev. B 66, 014407 (2002).

${ }^{15}$ D. Huertas-Hernando, Y. V. Nazarov, A. Brataas, and G. E. W. 
Bauer, Phys. Rev. B 62, 5700 (2000).

${ }^{16}$ T. Valet and A. Fert, Phys. Rev. B 48, 7099 (1993).

${ }^{17}$ W. Park, D. V. Baxter, S. Steenwyk, I. Moraru, J. W. P. Pratt, and J. Bass, Phys. Rev. B 62, 1178 (2000).

${ }^{18}$ G. E. W. Bauer, Y. Tserkovnyak, D. Huertas-Hernando, and A. Brataas, cond-mat/0205453 (unpublished).

${ }^{19}$ J. Bass and W. P. Pratt, J. Magn. Magn. Mater. 200, 274 (1999).

${ }^{20}$ K. Eid, D. Portner, R. Loloee, W. P. Pratt, and J. Bass, J. Magn. Magn. Mater. 224, L205 (2001).

${ }^{21}$ S. Zhang, P. M. Levy, and A. Fert, Phys. Rev. Lett. 88, 236601
(2002).

${ }^{22}$ K. M. Schep, J. B. A. N. van Hoof, P. J. Kelly, G. E. W. Bauer, and J. E. Inglesfield, Phys. Rev. B 56, 10805 (1997).

${ }^{23}$ C. J. Lambert and R. Raimondi, J. Phys.: Condens. Matter 10, 901 (1998).

${ }^{24}$ L. Giacomoni, B. Dieny, W. P. Pratt, Jr., R. Loloee, and M. Tsoi (unpublished).

${ }^{25}$ Y. Tserkovnyak and A. Brataas, Phys. Rev. B 64, 214402 (2001).

${ }^{26}$ H. Kurt, W.-C. Chiang, C. Ritz, K. Eid, W. P. Pratt, Jr., and J. Bass (unpublished). 